\title{
Demonstration of a chaos generator with two time delays
}

\author{
Min Won Lee, Laurent Larger, ${ }^{*}$ Vladimir Udaltsov, ${ }^{*}$ Éric Genin, and Jean-Pierre Goedgebuer* \\ Laboratorie d'Optique P.M. Duffieux, Centre National de la Recherche Scientifique Unité Mixte de Recherche 6603, Université de \\ Franche-Comté, Institut des Microtechniques, 25030 Besançon Cedex, France
}

\begin{abstract}
We demonstrate a chaos generator involving two time delays and two nonlinear functions. Dynamic behaviors are numerically and experimentally observed. The complexity of the dynamics is discussed in terms of Lyapunov exponents and dimensions. The setup can provide a new architecture for enhancing message security in chaos encryption systems.
\end{abstract}

Chaotic dynamics with a time delay have been studied for many years. In the past decade these dynamics have contributed to the development of optical systems in chaos synchronization ${ }^{1}$ and chaos anticipation. ${ }^{2}$ Some of these systems have been developed for chaotic message encryption and decryption ${ }^{3-5}$ because of their ability to mask a message inside high-complexity noiselike chaotic signals, as well as to recover the message at the receiver side with a synchronized chaotic transmitter and receiver. The confidentiality of such encryption systems typically depends on the complexity of the chaotic behaviors, on the number of possible parameters, producing such dynamics, and on the complexity of the system's architecture. Although chaos encryption provides strong security, it was recently reported that chaos encryption can be broken through some conventional time series analysis techniques in a cryptanalytic context. ${ }^{6,7}$ Thus it is of interest to enhance chaos encryption through any other technique. In this Letter we report and analyze an optoelectronic chaos generator involving two time delays and two nonlinear processes. This enhanced architecture should prevent chaos encryption from being broken or at least make it very difficult. The proposed setup is intended to strengthen chaos-based secure systems through the use of two or more time delays. Moreover, the study of such multiple delay dynamic systems meets the currently growing interest in various fields, such as the code-division multiple access communication technique, ${ }^{8}$ neural networks, ${ }^{9}$ and optical feedback systems. ${ }^{10}$

Recently, a chaos generator based on coherence modulation was reported, ${ }^{11}$ as was its application to optical encryption. ${ }^{12}$ With a similar physical principle based on optical coherence modulation we propose a chaotic generator with two time delays and two nonlinear functions. The results are shown in terms of an experimental bifurcation diagram and a numerical investigation of the Lyapunov exponents and dimensions.

The experimental setup is depicted in Fig. 1. A superluminescent diode is used as the light source, emitting at $\lambda_{0}=1.28 \mu \mathrm{m}$ with an output power of $P_{0}=650 \mu \mathrm{W}$ and a short coherence length $L_{c}=47 \mu \mathrm{m}$. An integrated unbalanced Mach-Zehnder (MZ) modulator yields an optical path difference (OPD) of $D_{0}=260 \mu \mathrm{m}$ that is much greater than the coher- ence length of the source. The MZ can be electrooptically modulated through the driving voltage with a half-wave voltage of $V_{\pi}=4.3 \mathrm{~V}$, thus performing the so-called coherence modulation [the MZ output consists of two wave packets separated by an OPD of $D=D_{0}+\delta D \gg L_{c}$, which cannot hence interfere]. The beam splitter divides the coherence-modulated beam into two arms. Each arm consists of an interferometer (INT1 and INT2, made by a bulk birefringent plate between crossed polarizers) with a static OPD ( $D_{1}$ and $D_{2}$, respectively) close to $D_{0}$ and a photodetector (PD1 and PD2, with photosensitivity of $K_{1}=1 \mathrm{~V} / \mu \mathrm{W}$ and $K_{2}=1.5 \mathrm{~V} / \mu \mathrm{W}$, respectively). The detected signals correspond to the respective intensity fluctuations, which are nonlinearly related to the electro-optic modulation of the MZ. The modulation transfer function is determined by INT1 and INT2, which perform a coherence demodulation: They both produce a half-contrast of two waves interference through the OPDs $\left(D_{1}-D\right)$ and $\left(D_{2}-D\right)$, which are smaller than $L_{c}$. Delay lines DL1 and DL2 electronically produce time delays of $T_{1}=512 \mu \mathrm{s}$ and $T_{2}=465.5 \mu \mathrm{s}$, respectively. The delayed nonlinear signals are then added and filtered by a low-pass filter, which limits the dynamics according to its response time $\tau=6.4 \mu \mathrm{s}$. The multiplier is used to amplify with a gain $G$ the resulting signal linearly with respect to an external voltage. Finally, the signal is fed back to serve as the electro-optic voltage for the coherence modulation, thus closing the oscillator loop of the nonlinear delayed dynamics. For details

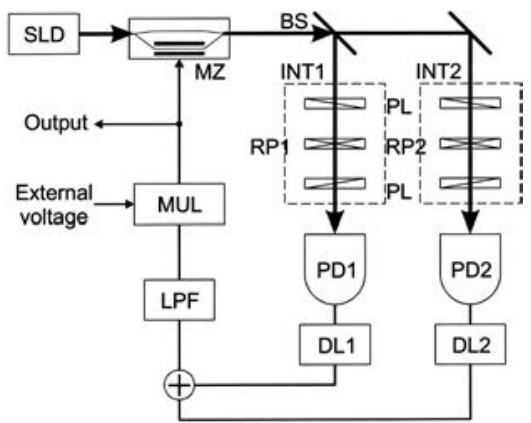

Fig. 1. Schematic experimental setup: SLD, superluminescent diode; BS, beam splitter; PLs, polarizers; RP1, RP2, retardation plates; LPF, low-pass filter; MUL, multiplier. 
on the chaos in coherence modulation the reader is referred to Ref. 11.

The feedback dynamics for this setup can be described by a delay differential equation with two time delays ${ }^{11}$ :

$$
\begin{aligned}
\delta D(t)+\tau \delta \dot{D}(t)= & F\left[\delta D\left(t-T_{1}\right), \beta_{1}, \Phi_{1}\right] \\
& +F\left[\delta D\left(t-T_{2}\right), \beta_{2}, \Phi_{2}\right],
\end{aligned}
$$

where the dynamic variable $\delta D(t)$ represents the OPD fluctuation; $F(D, \beta, \Phi)=\beta\left[1+(1 / 2) \cos \left(2 \pi \sigma_{0} D+\right.\right.$ $\Phi)]$ is the modulation transfer function resulting from the coherence demodulation; $\sigma_{0}=1 / \lambda_{0}$ is the wave number, $\beta_{1}=\alpha_{0} G K_{1} P_{0} / 8 \sigma_{0} V_{\pi}$ and $\beta_{2}=\alpha_{0} G K_{2} P_{0} / 8 \sigma_{0} V_{\pi}$ represent the weight of the nonlinear function; $\alpha_{0}=3.2 \times 10^{-3}$, which indicates $\simeq 24.9 \mathrm{~dB}$ optical loss of the system; and $\Phi_{1}=2 \pi \sigma_{0}\left(D_{0}-D_{1}\right)$ and $\Phi_{2}=2 \pi \sigma_{0}\left(D_{0}-D_{2}\right)$ are static phase shifts differentiating the two nonlinear functions produced by INT1 and INT2.

Because of the complexity of the system, the dynamic behavior can be explored with respect to many different parameters. For simplicity and to allow relevant comparison of the behaviors with respect to single delay systems, two cases are investigated: case $1, \Phi_{1}=$ $\pi / 2$ and $\Phi_{2}=0$, and case $2, \Phi_{1}=\pi$ and $\Phi_{2}=0$. Since the sensitivity ratio is $K_{2} / K_{1}=1.5$, the functions also exhibit different weight.

From Eq. (1) numerical simulations were performed with the fourth-order Runge-Kutta method with an integration step of $\mathrm{d} t=256 \mathrm{~ns}$. The numerical bifurcation diagrams obtained when increasing the gain $G$ ( $x$ axis) from 0 to 13 are reported in Fig. 2. The $y$ axis corresponds to the dynamic variable amplitude $(\delta D)$, and the gray scale represents the probability density of $\delta D$. Dark indicates a strong probability density of the dynamics.

Figure 2(a) shows the bifurcation diagram for case 1. As in the figure, the dynamics exhibit a global evolution from the fixed point to chaotic behaviors, but the route to chaos appears unconventional, without any clear period-doubling scenario. The first two bifurcations are Hopf-like; they remain in a previously observed situation called eye-bifurcation cascade (two successive direct and reverse supercritical Hopf bifurcations between a fixed point and a periodic oscilla$\operatorname{tion}^{11}$ at $G=3.1$ and $G=4.7$ ). Then, from the last fixed point, two close crises are observed between two chaotic like attractors $(G=4.8$ and $G=5.3)$. The system is then rendered in a chaotic state without any strong crisis from $G \simeq 5.3$, with a continuously growing amplitude and mean value with respect to $G$; the OPD wanders within a range of $\sim 15-\mu \mathrm{m}$ amplitude at $G=8.5$.

Likewise, the bifurcation diagram for case 2 is shown in Fig. 2(b). The scenario here is significantly different from the previous one, thus showing the strong influence of $\Phi_{i}$. The first bifurcation here is a crisis between a stable fixed point and a periodic oscillation with a large amplitude at $G=4.2$. A kind of period doubling is observed until $G=5.7$, which is interrupted by an unusual succession of periodic regimes. The system then jumps back to a fixed-point state at $G=6.2$ and rapidly renders chaotic from $G \simeq 6.3$. The evolution is then smooth, with chaos growing both in amplitude and mean value with respect to $G$. At $G=8.5$ the chaotic wandering range is the same as that in case 1 . Compared with the conventional route to chaos of single time delay systems, unusual bifurcation sequences are observed.

To investigate the complex increase of the dynamics with respect to the single time delay case, we calculated and compared the Lyapunov exponents and dimensions for the double time delay case. The reference single delay case corresponds to the setup in Fig. 1, for which $T_{1}$ and $T_{2}$ were adjusted to the same value $\left(T_{1}=T_{2}=512 \mu \mathrm{s}\right)$. The calculations were performed from the dynamics numerically obtained with $\Phi_{1}=\pi / 2, \Phi_{2}=0$, and $G=13$ for both cases. The method used for the Lyapunov exponents calculation was similar to that proposed by Farmer. ${ }^{13}$ In Fig. 3(a) the Lyapunov spectra are shown for both cases. The solid curve represents the spectra for the single time delay case and the dashed curve represents that for the dual time delay case. As seen in the figure, the number of positive Lyapunov exponents for the double time delay case is 54 , which is greater than the value corresponding to the single delay setup, 44 . Figure 3(b) represents the accumulated Lyapunov exponents deduced from Fig. 3(a). The solid curve refers to the single time delay case, and the dashed curve refers to the double time delay case. From this figure the Kaplan-Yorke dimension ${ }^{14}$ can be deduced through the maximum number of Lyapunov exponents leading to a positive value of the accumulated exponent curve. Dimensions of $D=109.3$ and $D=93.6$ were obtained for the double time delay case and for the single time delay case, respectively, thus confirming the increase of complexity in the dynamic behavior of the multiple delay situation. We also noted from Fig. 3(a) that the number of positive Lyapunov exponents is increased in

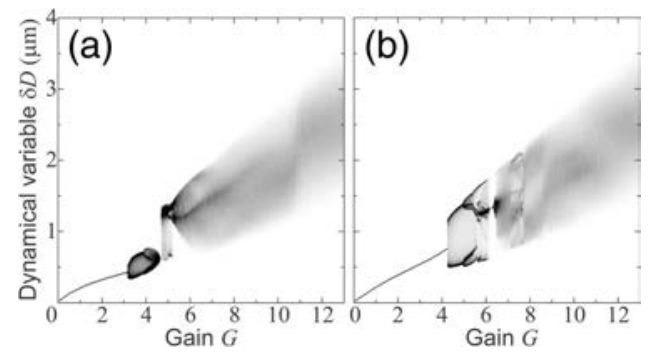

Fig. 2. Numerical bifurcation diagrams. (a) Case of $\Phi_{1}=\pi / 2$ and $\Phi_{2}=0$. (b) Case of $\Phi_{1}=\pi$ and $\Phi_{2}=0$.

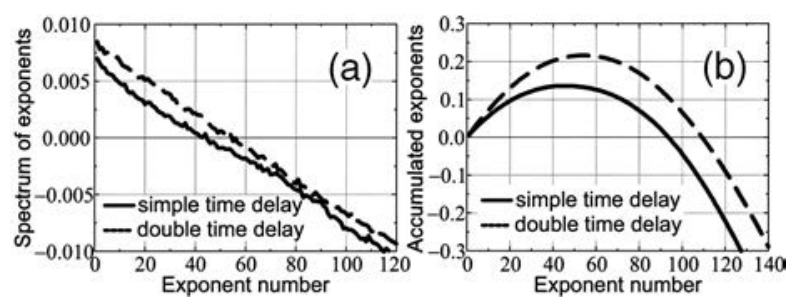

Fig. 3. Dynamic complexity for the double and single time delay. (a) Spectra of Lyapunov exponents. (b) Accumulated Lyapunov exponents. 


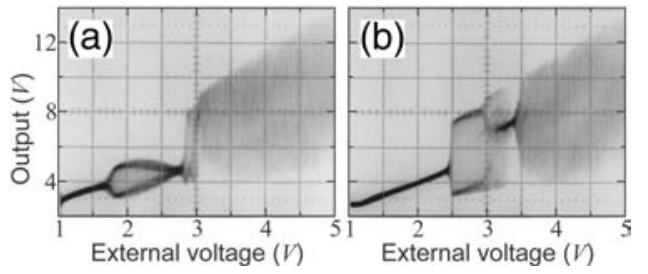

Fig. 4. Experimental bifurcation diagrams for the double time delay. (a) Case of $\Phi_{1}=\pi / 2$ and $\Phi_{2}=0$. (b) Case of $\Phi_{1}=\pi$ and $\Phi_{2}=0$.

Table 1. Comparison Between Experimental and Numerical Bifurcation Points ${ }^{a}$

\begin{tabular}{lcccc}
\hline & \multicolumn{4}{c}{ Bifurcation $^{a}$} \\
\cline { 2 - 5 } \multicolumn{1}{c}{ Gain $G$} & FP-P & P-FP & FP-C & C-C \\
\hline Numerical [Fig. 2(a)] & 3.1 & 4.7 & 4.8 & 5.3 \\
Experimental [Fig. 4(a)] & 2.3 & 4.7 & 4.9 & 5.4 \\
\hline
\end{tabular}

${ }^{a} \mathrm{FP}$, fixed point; P, periodic; C, chaos.

the dual delay case, and their magnitude is increased as well. This property is different from that observed in the single delay systems while increasing the time delay: The number of positive Lyapunov exponents is increased, but their magnitude is decreased. Hence, using two different time delays, $T_{1}$ and $T_{2}$, enhances complexity more than using the same delay. The proposed architecture appears to exhibit significant complexity improvement of its dynamic behavior.

Finally, the qualitative experimental behavior was explored and compared with the previous numerical investigations in terms of bifurcation diagrams. The experimental bifurcation diagrams were obtained by increasing the external voltage multiplier from 1 to $5 \mathrm{~V}$, which should correspond linearly to the numerical situation of $0.7 \leq G \leq 10.0$. Figure 4 shows the experimental bifurcation diagrams corresponding to the parameter values $\Phi$ considered in Fig. 2. Although the parameter values of the bifurcation points are recovered with only a relative precision, the successive bifurcations in both cases are observed in a similar way compared with the numerical diagrams [see comparison between Figs. 2(a) and 4(a) in Table 1]. The shape of the bifurcation diagrams is clearly recognized between Figs. 4 and 2, and we note that the chaotic regime amplitudes also correspond with good agreement to those obtained numerically: When the external voltage is $5 \mathrm{~V}$, the experimental chaotic output in both cases wanders within a range of $\sim 9-\mathrm{V}$ amplitude, which corresponds to $1.34-\mu \mathrm{m}$ OPD amplitude.

In conclusion, we have demonstrated a chaos generator based on coherence modulation techniques and its dynamics has been modeled by a nonlinear delay equa- tion with two time delays and two nonlinear functions. The numerical results conducted from that model show good qualitative agreement with the experimentally observed bifurcation situation under two different parameter settings. This agreement validates the established theoretical model. The numerical investigations from the dynamic model also show that the number of positive Lyapunov exponents and the values of Lyapunov exponents for the double time delay are greater than that for the single time delay. From the results it can be seen that the proposed system offers a higher complexity than with the conventional single delay system. The high complexity of such chaotic dynamics indicate that they could be advantageously used in chaos encryption techniques with enhanced message security.

This work was supported by the European Community through the Optical Chaos Communication Using Laser Transmitters project (IST-2000-29683). L. Larger's e-mail address is laurent.larger@univfcomte.fr.

*Also at Georgia Tech Lorraine-Centre National de la Recherche Scientifique Telecam, Unité Mixte de Recherche (UMR 6603), 2-3 Rue Marconi, 57070 Metz, France.

\section{References}

1. S. Sivaprakasam, and K. A. Shore, Opt. Lett. 24, 466 (1999).

2. S. Tang and J.-M. Liu, Phys. Rev. Lett. 90, 194101 (2003).

3. J.-P. Goedgebuer, L. Larger, and H. Porte, Phys. Rev. Lett. 80, 2249 (1998).

4. G. D. VanWiggeren and R. Roy, Phys. Rev. Lett. 81, 3547 (1998).

5. J.-M. Liu, H.-F. Chen, and S. Tang, IEEE J. Quantum Electron. 38, 1184 (2002).

6. R. Hegger, M. J. Bünner, and H. Kantz, Phys. Rev. Lett. 81, 558 (1998).

7. V. S. Udaltsov, J.-P. Goedgebuer, L. Larger, J.-B. Cuenot, P. Levy, and W. T. Rhodes, Phys. Lett. A 308, 54 (2003).

8. S. Penaud, J. Guittard, P. Bouysse, and R. Quéré, Electron. Lett. 36, 366 (2000).

9. J. Wei and S. Ruan, Physica D 130, 255 (1999).

10. T. Erneux, F. Rogister, A. Gavrielides, and V. Kovanis, Opt. Commun. 183, 467 (2000).

11. L. Larger, M. W. Lee, J.-P. Goedgebuer, W. Elflein, and T. Erneux, J. Opt. Soc. Am. B 18, 1063 (2001).

12. M. W. Lee, L. Larger, and J.-P. Goedgebuer, IEEE J. Quantum Electron. 39, 931 (2003).

13. J. D. Farmer, Physica D 4, 366 (1982).

14. J. Kaplan and J. Yorke, in Functional Differential Equations and Approximation of Fixed Points, H.-O. Peitgen and H.-O. Walther, eds. (SpringerVerlag, Berlin, 1979). 